\title{
An assessment of the accuracy of a panoramic radiograph as compared with cone-beam tomography in TMJ imaging
}

SADJ June 2017, Vol 72 no 5 p209 - p212

V Soni', B Buch²

\section{SUMMARY}

The articular disc of the temporomandibular joint (TMJ) cannot be seen on plain $\mathrm{x}$-ray examination but an accurate assessment of the space between the glenoid fossa and the head of the condyle may provide the clinician with a clue to a possible pathological problem. Measurements of the TMJ space seen on panoramic radiographs (PAN) and on cone beam computerised tomographic scans (CBCT) were carried out on films taken of of left and right TMJs of forty-six patients. On each image a vertical line was drawn digitally from the highest point of the condylar head to the glenoid fossa of the temporal bone. The lengths of the lines were recorded on Galileo software and compared using the Bland-Altman statistical test (total sample size 92 joints). Bias was achieved at mean differences of $1.03 \mathrm{~mm}$ with a $p$-value of $<0.001$ which was statistically significant. Left and right TMJ mean values showed a $0.09 \mathrm{~mm}$ difference. Age was insignificant at $p=0.40$. The Levels of Agreement indicated workable concord between the methods although a wide range was evident.

\section{INTRODUCTION}

The Temporomandibular joint (TMJ) is a synovial articulating cartilageous joint between the mandibular condyle and the glenoid fossa of the temporal bone. It is divided into upper and lower compartments by a fibro-cartilagenous articular disc. The upper joint compartment allows for protrusion, retraction and lateral movements whereas the lower joint compartment allows for hinge movements only, resulting in movements in the vertical dimension. ${ }^{1}$

1. Vishani Soni: $B C h D$ (Pret). Lecturer: Department of General Dental Practice, School of Oral Health Sciences, University of the Witwatersrand, Johannesburg.

2. Brian Buch: BSC (Hons) (Natal); SED (UCT); BDS Wits); MSc (Dent) (Wits). Emeritus Professor-University of Pretoria, Visiting Professor and Part-time Consultant: Division of Maxillo-Facial and Oral Radiology, Department of General Dental Practice, School of Oral Health Sciences, University of the Witwatersrand, Johannesburg.

Corresponding author

Vishani Soni:

Tel: 0114884854 E-mail: Vishani.Soni@wits.ac.za

\section{ACRONYMS}

CBCT: cone beam computerised tomographic

IQR: Inter-quartile Range

LoA: Limits of Agreement

PAN: panoramic radiograph

TMJ: temporomandibular joint

All of these mandibular manouevers may occur during mastication and are regulated by a combination of neurological control mechanisms together with complex muscle systems (muscles of mastication) that react to the nervous stimuli. However, limitations in mobility can be caused by muscular dysfunction or restricted movement of the articular disc which may constrain certain TMJ movements and may subsequently lead to TMJ dysfunction. ${ }^{2}$ The position of the articular disc cannot be seen on plain $x$-ray examination, eg. a panoramic radiograph, but an accurate assessment of the space between the glenoid fossa and the head of the condyle may provide the clinician with a clue to a possible pathological problem. ${ }^{3}$

Causes of TMJ dysfunction may be physiological, psychological or pathological. Physiological conditions which may be associated include excessive occlusal forces; malpositioned teeth due to crowding; early exfoliation or extraction of primary teeth resulting in a bite of convenience or an incorrect vertical or horizontal jaw relationship., ${ }^{4,5}$

Psychological conditions include sleep apnoea; psychosocial distress; environmental stress; bruxism and poor control of chronic TMJ pain. ${ }^{6-9}$ Pathological conditions that influence TMJ movement are osteo- arthritis of juveniles and adults; trauma such as motor vehicle accidents; benign and malignant lesions and cysts within the joints. ${ }^{10-12}$ In order to assess any of these conditions an accurate diagnosis is essential.

Radiographic interpretation is an important diagnostic tool in the evaluation of TMJ dysfunctions to identify bony changes or space deficiencies between the head of the 
condyle and the glenoid fossa. ${ }^{13,14}$ Several studies have reported on the accuracy of cone-beam tomography (CBCT) in the examination of the TMJ, but few have evaluated the use of panoramic radiographs (Pans) for assessment of the TMJ space. A study in 1980 stated that "due to magnification, lack of definition and superimposition of structures, panographs may be diagnostically inferior to intra-oral radiography". ${ }^{15}$ Nevertheless Pans still appear to be widely used for diagnosis of TMJ space deficiencies and bone disorders. ${ }^{16-18}$ Since 1982, digital radiography has enabled the electronic storage of panoramic images for easy recall.

In 1998 an innovation, the three-dimensional cone-beam computerised tomogram, (CBCT) was introduced. The technique provided the practitioner with images at various depths within the tissue, unimpeded by superimposed structures. ${ }^{17}$ CBCT provided surface and sectional information about the craniofacial structures. ${ }^{19}$ Such equipment, however, is extremely costly and moreover should not be utilised as a routine modality. For this reason the average dentist relies on a Pan. Despite certain flaws, e.g. superimposition of structures, Pans are still currently being used to diagnose bony changes and joint space deficiencies and have allowed generally correct interpretation of the bony components of TMJ. ${ }^{16}$ The question may be raised, however, as to what are the levels of agreement between the data measured on a Pan with that measured on the purportedly technically superior CBCT projection.

This study specifically aimed to determine the extent of agreement between data derived from Panoramic radiographs and CBCT scans of the TMJ in determining the height of the space between the head of the mandibular condyle and the glenoid fossa of the temporal bone.

\section{MATERIALS AND METHODS}

The number of radiographs taken at the Radiology section of the Wits School of Oral Health Sciences includes approximately 50 Pans per day and about 10 CBCT scans per week. This routine provided sufficient material for the current project. From the archives of the Radiology Section, the records of sixty three patients who had been subjected to both a Pan and a CBCT scan between March and July 2015 inclusive were randomly selected, using the Excel random number selection programme, and were assessed for inclusion in the study. All radiographic views were required to be of highly acceptable quality. Seventeen cases were excluded from the study for reasons of the patient having undergone condylar fusion, condylectomy, condylar prosthesis or another form of condylar pathosis. Hence a total of 92 radiographs of joint spaces were included. Each radiograph demonstrated completely discernible TMJ spaces enabling accurate measurement on both left and right sides. The joint space was defined by a digitally drawn vertical line from the highest point of the condylar head to the glenoid fossa of the temporal bone for each radiograph (Figure 1). The standardized measuring gauge included in the Galileo Gax 5 software (Sirona Dental, SA) of the Galileos Cone-Beam scanner was used in the determination of all measurements. The Pans were assessed within their fixed focal

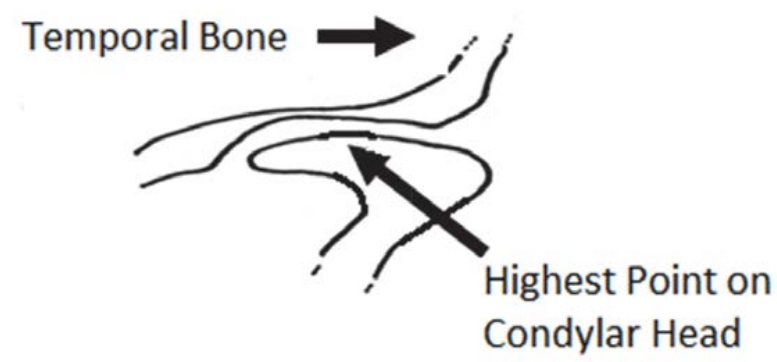

Figure 1: Measurement of TMJ space in cross-sectional view of CBCT.

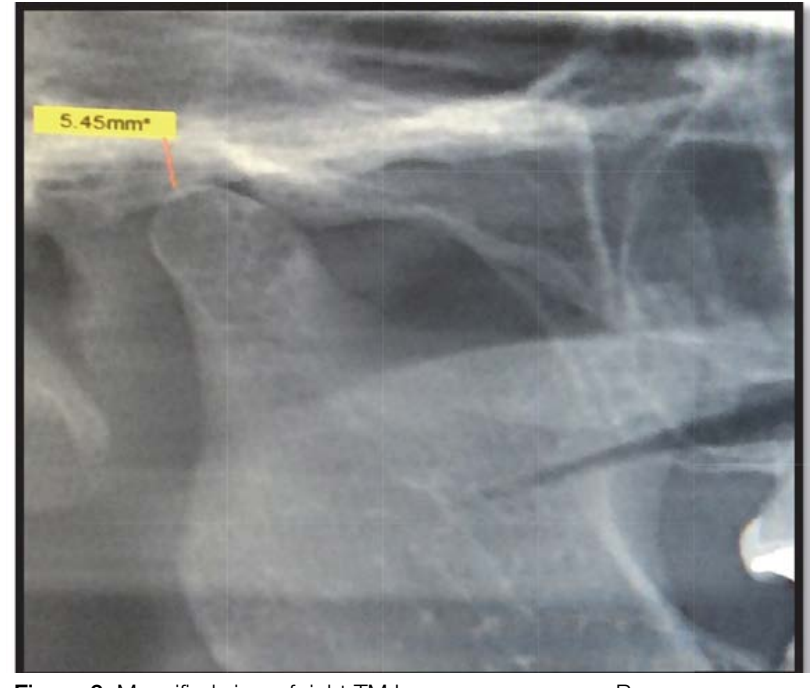

Figure 2: Magnified view of right TMJ space as seen on a Pan.

troughs (Figure 2) while the CBCTs were assessed at the deepest levels of their tangential views with the patient in the correct occlusal position, having closed on a standardized bite stick. Each CBCT was further viewed in the cross-sectional mode in order to ensure that the vertical dimensions of the tangential mode corresponded to those of the cross-sectional views (Figure 3). The measurements were performed by one researcher in the first instance and $20 \%$ were repeated without collusion by a second.

The data were recorded electronically and were analyzed using the Bland-Altman method for assessing agreement between two methods of clinical measurement. ${ }^{20}$

The effect of age and sex on the bias between the methods was determined by regressing the between-method differences on patient age and sex.

Data analysis was carried out using SAS (version 9.4 for Windows). The 5\% significance level was used.

\section{RESULTS}

The final sample of 46 patients was aged between 10 and 68 years (median 29 years) and 63\% were male. The inter-observer agreement between the two sets of data was found to be $89 \%$.

\begin{tabular}{c|c|c|c|c|c|c|c}
\multicolumn{6}{c}{ Table 1: Descriptive data analysis of overall data set } \\
Variable & Units & N & Median & Interquartile range & Minimum & Maximum \\
\hline Age & years & 46 & 29 & 20 & 42 & 10 & 68 \\
PAN & $\mathrm{mm}$ & 92 & 3,03 & 2,20 & 4,92 & 0,78 & 10,00 \\
CBCT & $\mathrm{mm}$ & 92 & 4,45 & 3,44 & 5,76 & 1,49 & 10,58
\end{tabular}




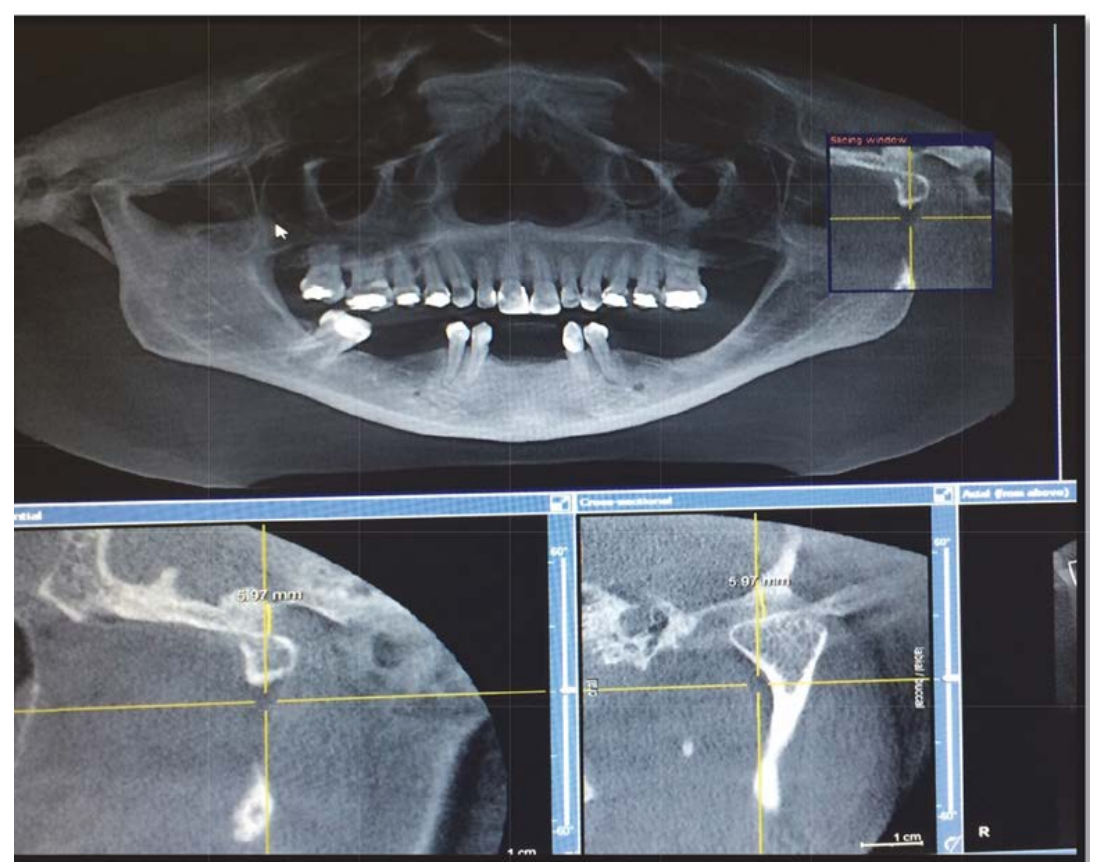

Figure 3: Measurement of the TMJ space on sagittal and cross sectional views on CBCT scan. This case shows full agreement of the measurement at $5.97 \mathrm{~mm}$..

\section{Bland-Altman plot}

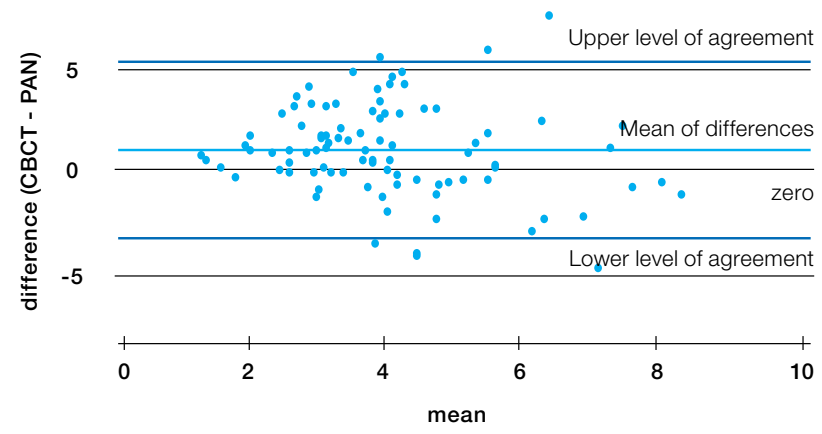

Figure 4: Bland-Altman plot of the difference between the CBCT and PAN measurements against the mean of the two measurements.

Perhaps the immediately striking feature displayed by the descriptive statistics was the variation in the height of the joint space, for this ranged from around $1 \mathrm{~mm}$ right up to over $10 \mathrm{~mm}$ (Table 1). In general the CBCT measurements were greater than the corresponding Pan data (Table 1).

The Bland-Altman plot of the difference between the CBCT and PAN measurements against the mean of the two measurements is shown in Figure 4. The calculated bias of $1.03 \mathrm{~mm}$ differed significantly from zero $(p<0.0001)$. The lower and upper Levels of Agreement were determined at -3.20 and 5.25 respectively. Hence, results measured by $\mathrm{CBCT}$ may at the extreme statistical agreement levels be $5.25 \mathrm{~mm}$ above the measurements made on Pans or $3.20 \mathrm{~mm}$ below. The bias did not increase or decrease with increasing mean space gap, as determined by a regression of the differences on the means $(p=0.097)$. The effect of age and sex on the bias was not significant ( $p=0.40$ and 0.17 respectively).

A priori limits of agreement of -1.5 to $1.5 \mathrm{~mm}$ were set at the outset, determined on the basis of biological and clinical criteria. Only $54 \%$ of the data points fall within these LoAs.

\section{DISCUSSION}

The ideal position of the mandibular condyle within the glenoid fossa is often influenced by the dimensions of the TMJ space. ${ }^{3}$ Prior research has related the dimensions of TMJ space, joint morphology and joint pathology to dental occlusion and positional variations of the condyle within the joint. ${ }^{8,19-}$ 22,24,26-29 Pans have commonly been used to provide an initial assessment of these situations, with minimal limitations. ${ }^{26,27,29}$ Hence, Pans remain the prime TMJ diagnostic modality. The current study set out to investigate whether measurements of the joint space made on Pans were of a validity commensurate with those achieved by CBCT scans, a technological advance.

Although the TMJ space, assessed anatomically, has been shown to vary with age and gender, ${ }^{23,25}$ these relationships were not shown in this study to have an effect on the differences between the two methods. However, there was variation in the measurements recorded from the different radiographs, as has been found in previous studies. ${ }^{13,21,22}$ The Bland and Altman analysis revealed a relatively small bias ( the mean of the differences between the techniques) but this was associated with statistically determined Levels of Agreement which were quite widely separated, and in fact exceeded the ranges reported from other studies. ${ }^{21-23} \mathrm{~A}$ small bias but wide limits of agreement may indicate that whilst the average accuracy of the measurements may be reasonable, the data is too variable to be useful. Consider that the actual measurements of the joint space ranged from 0.78 to $10.58 \mathrm{~mm}$. Yet the statistically determined Limits of Agreement extended from -3.20 to $5.25 \mathrm{~mm}$, too wide a range to be clinically precise.

Bland and Altman recommend that Limits of Agreement be set at the outset of these comparisons, an a priori exercise based on biological and clinical criteria. Applying the Limits of Agreement selected a priori in this study (+ $1,5$ and $-1,5)$ showed a lack of agreement between the radiological methods when reasonable clinical expectations are taken into account.

\section{CONCLUSION}

The two radiological methods assessed in the study have not been shown to be interchangeable when precision measurements of the joint space are required. Whilst CBCT scans may be the first choice of clinicians, the Panoramic radiograph nevertheless offers data which may be useful in the initial diagnostic exercise, provided that interpretation is done with caution. The elevated cost and radiation dose associated with CBCT scanning mitigate against its routine usage.

\section{LIMITATIONS OF THE STUDY}

No assessment of the repeatability of the measurements was done. Joint space was assessed in only one dimension, whereas considerable variation could have occurred in other aspects. 
Declaration: Neither of the investigators hold any interest in either of the radiation units investigated.

\section{Acknowledgments}

The researcher would like to thank the Ethics Committee for allowing the research to be conducted at the Charlotte Maxeke Academic Hospital. Grateful appreciation is recorded to Dr Petra Gaylard of Data Management and Statistical Analysis, University of the Witwatersrand, Johannesburg.

\section{References}

1. Moore KL, Dalley AF and Agur AM. Clinically Oriented Anatomy, 7th ed. USA Philadelphia: Lippincott Williams \& Wilkins, 2013:914-6.

2. Kuwahara T, Miyauchi S, Maruyama T. Clinical classification of the patterns of mandibular movements during mastication in subjects with TMJ disorders. Int J Prosthodont. 1992;5:122-9.

3. Markic G, Müller L, Patcas R, Roos M, Lochbühler N, Peltomäki T, Karlo CA, Ullrich O, Kellenberger CJ. Assessing the length of the mandibular ramus and the condylar process: a comparison of OPG, CBCT, CT, MRI, and lateral cephalometric measurements. Eur J Orthodont.2015;37:13-21.

4. Howe RP, McNamara JA, O'Connor KA. An examination of dental crowding and its relationship to tooth size and arch dimension. Am J Orthod. 1983;83:363-73.

5. Lee J, Kim YI, Hwang DS, Kim KB, Park SB. Effect of occlusal vertical dimension changes on postsurgical skeletal changes in a surgery-first approach for skeletal Class III deformities. Am J of Orthod and Dentofacial Orthoped. 2014;146:612-9.

6. Fillingim RB, Ohrbach R, Greenspan JD, Knott C, Dubner R, Bair E, Maixner W. Potential psychosocial risk factors for chronic TMD: descriptive data and empirically identified domains from the OPPERA case-control study. $J$ of Pain. 2012;12: T46-T60.

7. Lei J, Liu MQ, Yap AU, Fu KY. Sleep disturbance and psychologic distress: prevalence and risk indicators for temporomandibular disorders in a Chinese population. J Oral Facial Pain Headache. 2015;29:24-30.

8. Trenouth MJ. The relationship between bruxism and temporomandibular joint dysfunction as shown by computer analysis of nocturnal tooth contact patterns. J of Oral Rehab.1979; 6:81-7.

9. Xu J, Long X, Cheng AHA, Cai H, Deng M, Meng Q. Modified condylar distraction osteogenesis via single pre-auricular incision for treatment of temporomandibular joint ankylosis. J Craniofacial Surg. 2015;26:509-11.

10. Kopp S, Nilner M, Petersson A, Rohlin M. Relationship between clinical and radiologic findings of the temporomandibular joint in rheumatoid arthritis. Oral Surg Oral Med Oral Pathol. 1988;66:639-43.

11. Yücel E, Börkan Ü, Mollaoglu N, Erkmen E, Günhan Ö. Histological evaluation of changes in the temporomandibular joint after direct and indirect trauma: an experimental study. Dent Traumatol. 2002;18: 212-6.

12. Zwir LM, Terreri MT, Sousa SA, Fernandes AR, Guimarães AS, Hilário MO. Are temporomandibular joint signs and symptoms associated with magnetic resonance imaging findings in juvenile idiopathic arthritis patients? A longitudinal study. Clin. Rheumatol. 2015;34:2057-63.

13. Al-koshab M, Nambiar P, John J. Assessment of condyle and glenoid fossa morphology using CBCT in South-East Asians. PloS one. 2015;10:e0121682.

14. De Boer EW, Dijkstra PU, Stegenga B, de Bont LG, Spijkervet FK. Value of cone-beam computed tomography in the process of diagnosis and management of disorders of the temporomandibular joint. Br J Oral Maxillofac Surg. 2014;52:241-6.

15. Alattar MM, Baughman RA, Collett WK. A survey of panoramic radiographs for evaluation of normal and pathologic findings. Oral Surg Oral Med Oral Pathol. 1980; 50:472-8.

16. Ladeira DB, da Cruz AD, Almeida SM. Digital panoramic radiography for diagnosis of the temporomandibular joint:
CBCT as the gold standard. Braz Oral Res.2015;29: S180683242015000100303

17. Quantrill JR , Lewis JES. The interpretation of temporomandibular joint radiographs. S Afr Med J. 1974;48:1905-12.

18. Yousefian J, Trimble D, Folkman G. A new look at the treatment of Class II Division 2 malocclusions. Am J of Orthod Dentofacial Orthopeds. 2006;130:771-8.

19. Kapila SD, Nervina JM. CBCT in orthodontics: assessment of treatment outcomes and indications for its use. Dentomaxillofac Radiol. 2015;44:p.20140282.

20. Bland JM, Altman DG. Statistical method for assessing agreement between two methods of clinical measurement. Lancet. 1986;327: 307-10. 10. 1016/SO140-6736(86)90837-8 (Pub Med)

21. Pullinger AG, Solberg WK, Hollender L, Guichet D. Tomographic analysis of mandibular condyle position in diagnostic subgroups of temporomandibular disorders. J

22. Dalili Z, Khaki N, Kia SJ, Salamat F. Assessing joint space and condylar position in the people with normal function of temporomandibular joint with cone-beam computed tomography. Dent Res J.2012; 9:607-12.

23. Kinniburgh RD, Major PW, Nebbe B, West K, Glover KE. Osseous morphology and spatial relationships of the temporomandibular joint: comparisons of normal and anterior disc positions. Angle Orthod. 2000;70:70-80.

24. Martins E, Silva JC, Pires CA, Ponces-Ramalhão MJF, Lopes JD. Coronal joint spaces of the temporomandibular joint: Systematic review and meta-analysis. J Clin.Exp Dent. 2015;7:e435-40.

25. Ikeda K, Kawamura A, Ikeda R. Assessment of optimal condylar position in the coronal and axial planes with limited conebeam computed tomography. J Prosthod. 2011;20:432-8.

26. Lins CC, Tavares RMC, da Silva CC. Use of digital panoramic radiographs in the study of styloid process elongation. Anat Res Int. 2015;2015:474615.

27. Winocur E, Reiter S, Krichmer M, Kaffe I. Classifying degenerative joint disease by the RDC/TMD and by panoramic imaging: a retrospective analysis. J Oral Rehabil. 2010;37:171-7.

28. Kaur B, Sehgal R, Logani A, Dhar P. Morphometric evaluation of temporomandibular joint using cone beam computer tomography. Am J Oral Med Radiol. 2015;2:169-76.

29. Sinha VP, Pradhan H, Gupta H, Mohammad S, Singh RK, Mehrotra D, Pant MC, Pradhan R. Efficacy of plain radiographs, CT scan, MRI and ultra sonography in temporomandibular joint disorders. Natl J Maxillofac Surg. 2012;3:2-9. 